\title{
Telencephalic Lesions and Behavior in the Teleost, Macropodus opercularis: Reproduction, Startle Reaction, and Operant Behavior in the Male
}

\author{
ROGER E. DAVIS, 1 JEFFREY KASSEL, \\ and PATRICIA SCHWAGMEYER \\ Mental Health Resarch Institute \\ and Neuroscience Laboratory, \\ University of Michigan, \\ Ann Arbor, Michigan 48109
}

\begin{abstract}
Bilateral ablation of the telecephalon at the level of the anterior commissure blocked reproduction in male paradise fish. Lesioned males performed species typical displays at the start of the spawning trial but thereafter avoided the female, and they did not build nests. All the sham-operated males spawned and built nests. The reaction of the male to a startle stimulus and the rate of operant responding for visual reinforcement were used as additional, independent measures of the behavioral effects of the ablation. Removal of the telencephalon greatly increased startle reactivity and decreased the rate of operant responding. Hyperreactivity could account for long response latencies, reduced mobility, and other reported effects of telencephalic ablation in teleosts which have previously been attributed to impaired nonspecific arousal.
\end{abstract}

\section{INTRODUCTION}

The effects on reproductive behavior of removal of the telencephalic hemispheres have been investigated in a number of teleosts (for reviews, see Healy, 1957; Segaar, 1965; Aronson and Kaplan, 1968; Bernstein, 1969). Noble $(1936,1937)$ and Noble and Borne (1941) reported that telencephalic ablations impair or obliterate reproductive behavior of two cichlids, Tilapia macrocephala and Hemichromis bimaculatus, and a belontid, Betta splendens. However, their experimental procedure, and the exact nature of the behavioral deficits and of the brain tissue ablations are ambiguous or not described.

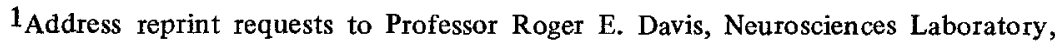
The University of Michigan, Ann Arbor, Michigan 48109. 
Other investigations in Tilapia (Aronson, 1948; Overmier and Gross, 1974) and another cichlid, Hemihaplochromis philander (Ribbink, 1972), confirmed that reproductive behaviors are greatly decreased following telencephalonectomy. Some fertile matings were seen. Reproduction is also decreased in the guppy, Poecilia reticulatus, and the platyfish, Xiphophorous maculatus, with the telencephalon removed, but fertile matings continue to occur (Segaar, 1965; Kamrin and Aronson, 1954). In male sticklebacks (Gasterosteus aculeatus) courtship and nestfanning behaviors are disrupted by ablation of different areas of the telencephalon (Segaar and Nieuwenhuys, 1963).

Preliminary investigations in our laboratory revealed that ablation of the telencephalon anterior and dorsal to the preoptic nucleus blocks reproductive behavior in male Macropodus which are paired with intact females. This agrees with results obtained with Betta by Noble and Borne (1941) but not by Noble (1936). Betta and Macropodus are in the same subfamily, Macropodinae (Liem, 1963), and they have similar courtship, mating, and parental behaviors, suggesting that the role of the telencephalon in sociosexual behaviors may be similar. The present paper describes the effects of bilateral extirpation of the telencephalon on reproduction and other behavioral systems in male Macropodus.

Reproduction might be blocked by disturbances of sensory, motor, and integrative processes including the neural and endocrine mechanisms of reproductive behavior. Courtship and mating consists of intensive, coordinated behavior by the male-female pair which may place a heavy demand on a variety of behavioral systems. Though telencephalic lesions and ablations have minor effects on posture, locomotion, and general maintenance behaviors in teleosts (Segaar, 1965), and defects may be variable and difficult to measure, objective analysis of ablation-induced changes in nonreproductive behaviors may provide insights into how reproduction is distupted and, more generally, the function of the telencephalon.

One behavioral variable which could affect the outcome of a social encounter is the fish's reactivity to external stimulation (Davis, 1975). We investigated whether telencephalon ablation alters reactivity to a startle stimulus. Another variable is the capacity of the fish to perform behaviors entailing specific patterns of locomotion and orientation to visual cues, which we assessed with operant conditioning methods (Davis, Harris, and Shelby, 1974; Davis, Mitchell, and Dolson, 1975). The fish received repeated operant sessions before and after removal of the telencephalon, interspersed with a startle reaction trial and a 10 -day spawning trial.

\section{METHOD}

\section{Subjects}

Adult male paradise fish, Macropodus opercularis (L.), $\bar{x}=5.1 \mathrm{~cm}$ body length and $\bar{x}=3.9 \mathrm{~g}$ body weight were obtained from domesticated stocks in 

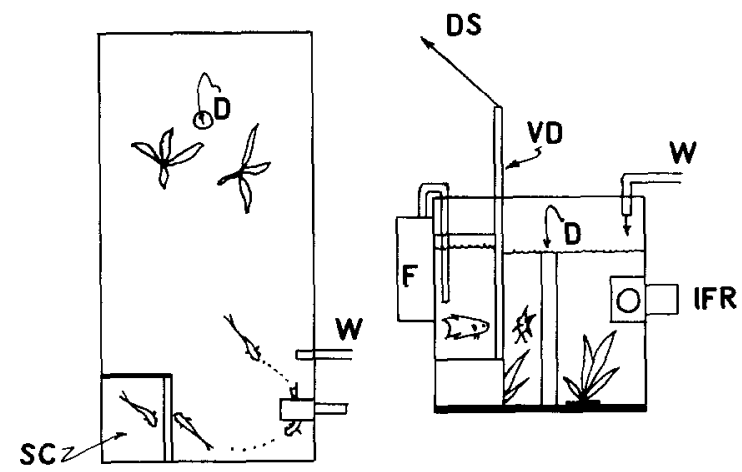

Fig. 1. Operant tank as seen from above (left) and from the front end. When the subject swam through the manipulandum ring, the door to the stimulus compartment was raised, allowing the subject to see the stimulus fish through a clear glass window for 30 sec. D, water drain; DS, drawstring operated by linear motor (not shown); F, water filter: IFR, infrared photodetector; SC, stimulus fish compartment; VD, vertical door; W, water supply $(400 \mathrm{ml} / \mathrm{min})$.

Florida. The daily cycle of diffuse natural light in the laboratory was augmented by a 14:10-hr, L:D cycle of "daylight" fluorescent light. The fish received Tetramin staple conditioning food and frozen brine shrimp in one or two daily feedings. The fish were placed in individual isolation tanks (Davis $e t$ al., 1974) for 1 to 2 weeks to screen subjects for readiness to respond to social stimulus reinforcement. Temperatures were 24 to $26^{\circ} \mathrm{C}$.

\section{Operant Tank}

The apparatus described in Davis et al. (1975), slightly modified, was used. The front end of the tank was kept clear to observe the fish. The opposite end and the sides were overgrown with algae. The stimulus fish compartment, manipulandum, and water inlet were placed at the front of the tank; the water outlet was at the rear (Fig. 1).

The stimulus compartment consisted of a rectangular, clear glass jar, $8 \mathrm{X}$ $13 \times 20-\mathrm{cm}$ tall which was placed in the front left-hand corner of the tank behind an opaque screen containing a vertical sliding door. Raising the door revealed a $12 \times 14-\mathrm{cm}$ window through which the subject could view the entire stimulus compartment. The investigator could see the stimulus fish and the subject simultaneously through the front of the tank. The water in the stimulus compartment was continuously circulated through an external filter. There was no exchange of water between the compartment and the tank.

The swim-through manipulandum consisted of an infrared beam across a $2.5 \mathrm{~cm}$ ring (Davis et al., 1975). The ring was attached to the tank wall $19 \mathrm{~cm}$ 
from the stimulus compartment door (Fig. 1). The infrared photodetector was placed outside the tank. The beam passed through the tank wall and a slit in the ring, across the ring to a mirror which was masked with a red filter, and back across the ring to the photodetector. When the fish interrupted the beam, the stimulus compartment door was opened for $30 \mathrm{sec}$. During this period, the subject and stimulus fish could see and respond to each other.

The photodetector was interfaced to a PDP-8F digital computer which registered response frequency, the duration which the fish remained in the beam, presented the visual reinforcements, and compiled the data. Responses made during a 30 -sec reinforcement period were not reinforced and were tallied separately from reinforced responses.

\section{PROCEDURE}

\section{Preoperative Operant Conditioning}

Thirteen males were screened for readiness to perform the swim-through response when rewarded by a $30-\mathrm{sec}$ view of a stimulus male. The male was placed in the operant tank for 18 to $24 \mathrm{hr}$ on a schedule of continuous reinforcement (CRF). The session was started with a 30-sec free reinforcement. The door was opened when the subject first approached the stimulus fish, then closed $30 \mathrm{sec}$ later. Fish which performed 10 or more swim-through reponses per hour for at least $3 \mathrm{hr}$ were selected as subjects. The subject subsequently received three 1 -hr sessions, at intervals of 2 to 3 days. The mean rate of responding in the three sessions was used as the preoperative behavioral baseline. The day following the final session, eight of the males were telencephalon-ablated (tele- $x$ ) and five received a sham operation. During this period, opaque partitions between the isolation tanks prevented the individual from seeing other fish. One tele- $x$ male was discarded when the postmortem examination revealed that only one hemisphere had been removed.

\section{Surgery}

The telencephalon was removed by aspiration through a 2-mm hole which was cut in the cranium with a trephine. The trephine was clamped in a high speed drill. The fish was anesthetized in $0.04 \%$ Finquel (Ayerst) and held in a wet paper towel. The hole was cut in the midline between the orbits. The disc of bone, which was usually only partially freed by the trephine, was removed with a forceps. The lobes of the telencephalon were aspirated individually through a 22-gauge flat-tipped syringe needle. The hole was covered by replacing the disc of bone or with a daub of white petroleum jelly. In the sham operation, the hole was cut in the cranium, the telencephalon was visualized, and then the hole was immediately covered. The fish were allowed 
to recover to an upright position prior to being returned to the isolation tanks. The day of surgery was designated as Day 1 of the experiment.

\section{Postoperative Treatments}

When the males returned to isolation following surgery, the opaque partitions between the tanks were removed so that neighboring fish could see one another. The purpose of this procedure was to decrease responsivity to external stimulation, including social stimulation, which increases in socially isolated males (Davis, 1975).

Visual Reinforcement Sessions (Days 2 to 6)

On Days 2, 4, and 6 the male was placed in the operant tank, given a 30-sec free reinforcement and then allowed to respond on a CRF schedule for $1 \mathrm{hr}$.

\section{Startle Reaction Trial (Day 8)}

The males' reaction to a combined aural and visual startle stimulus was measured in a neutral tank as previously described (Davis, 1975). The rates of swimming and air gulping were measured for $5 \mathrm{~min}$ immediately preceding and following the startle stimulus. The magnitude of the reaction was determined as the percentage of suppression of swimming and breathing $(100 \times 1$ minus (poststimulus rate/prestimulus rate)).

\section{Spawning Trial (Days 9 to 19)}

The male was placed in a 9-liter tank with a female until they spawned or for a maximum of 10 consecutive days. The female was put in the tank the day before the male. The frequencies of approach, lateral and frontal displays, quivers, and attacks (Davis and Kassel, 1975) by the male were recorded during the first $10 \mathrm{~min}$ of the trial. The pair was observed daily to determine the time of spawning.

\section{Postspawning Visual Reinforcement Trials (Days 19 to 22)}

On Day 19, the male was placed in the operant tank for a 1-hr session on CRF, with a male visual stimulus, following an initial, 30-sec free reinforcement. Following the session, the opaque partition between the isolation tanks was replaced. Social stimulus deprivation was instituted to possibly increase the male's responsivity (Davis, 1975). Forty-eight hours later, the male's responses for visual reinforcement were measured in a 24-hr session of operant responding on a CRF schedule. The session was started with a 2-min free reinforcement. 


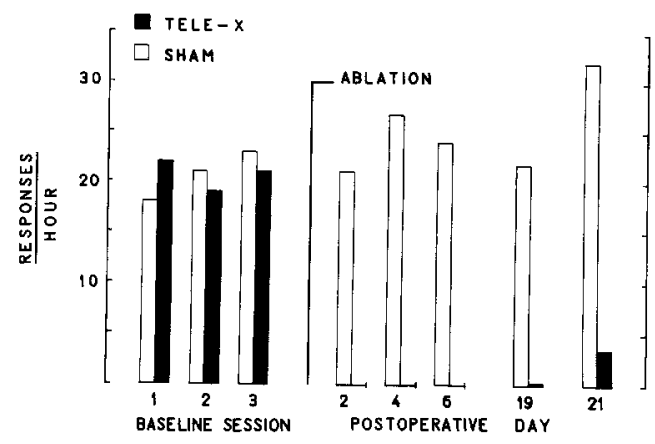

Fig. 2. The mean rate of operant responding in 1-hr sessions prior to and following bilateral removal of the telencephalon. The session on Day 21 was $24 \mathrm{hr}$ in duration (see text), the data shown are for the first hour.

\section{Histology}

On Day 23, the male was killed in ice water and the brain was prepared for histology. In half of the males, the brain was fixed in formalin, then embedded in albumin, and cut in $8-\mu \mathrm{m}$, parasaggital sections in a freezing microtome. In the other males, the entire head was fixed for $48 \mathrm{hr}$, decalcified, embedded in paraffin, and cut in $8-\mu \mathrm{m}$ coronal sections. The sections were stained with hematoxylin and eosin. The extent of the ablations was determined by inspection of serial brain sections of the tele-x and sham-operated males.

\section{RESULTS}

\section{Operant Responding}

Prior to the ablation, the sham and tele-x groups showed no significant difference in mean response rate (Fig. 2). Following surgery, the sham group continued to respond, with no marked change in rate, on Days 2, 4, 6, and 19. The tele-x group, on the other hand, made no responses on Days 2, 4, and 6 , and one male made one response on Day 19.

In the 24-hr session starting on Day 21 , the response rate of the sham group was high during the first hour, decreased over the next several hours, and then remained relatively uniform for the rest of the session (Fig 3). The mean response rate in the first hour, $32 / \mathrm{hr}$, was not significantly higher than the rate in the preceding 1-hr session on Day 19, 22/hr, (Fig. 2). Thus, the hypothesis that the stimulus deprivation from Days 19 to 21 would increase the response rate was not upheld. 


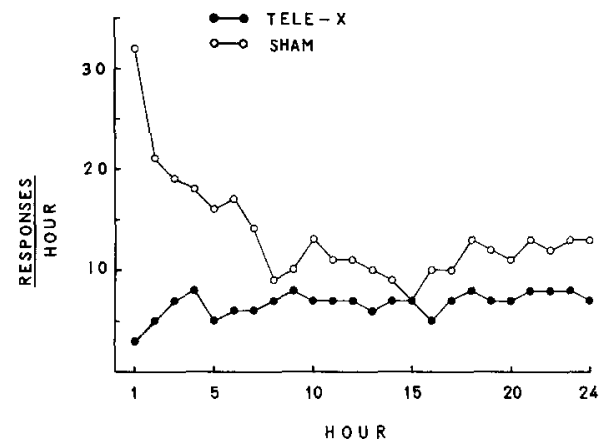

Fig. 3. The mean hourly response rate during the 24-hr session, Days 21 to 22 . The session started at $1200 \mathrm{hr}$. The operant tank was continuously illuminated.

The tele-x group showed a low, uniform response rate throughout the 24-hr session (Fig. 3). The tele- $x$ and sham groups differed in rate only in the first $2 \mathrm{hr}$ of the session. While all the shams responded during the session, only four of the six tele-x males responded. In the first $2 \mathrm{hr}$, only two of the six tele-x males responded.

\section{Startle Reaction}

During the 5-min period prior to presentation of the startle stimulus, the tele-x group crossed significantly fewer lines of the activity grid than the shams (Table 1); the difference in mean air-gulp frequency during that period was not significant. In addition to being less active before the startle stimulus, the tele-x group showed a greater suppression of air gulp and of swimming following the startle stimulus than the shams. All of the shams resumed

\section{TABLE 1}

Suppression of Swimming and Air-Gulp Frequency Following a Startle Stimulus

\begin{tabular}{lcccccc}
\hline & \multicolumn{2}{c}{ Air-gulp frequency } & & \multicolumn{2}{c}{ Swimming } & \\
& $N$ & $\begin{array}{c}\text { Prestartle } \\
\text { period } \\
(5 \mathrm{~min})\end{array}$ & $\begin{array}{c}\text { Suppression } \\
(\%)\end{array}$ & $\begin{array}{c}\text { Prestartle } \\
\text { period } \\
(5 \mathrm{~min})\end{array}$ & $\begin{array}{c}\text { Suppression } \\
(\%)\end{array}$ & $\begin{array}{c}\text { Post-startle } \\
\text { immobility } \\
(\mathrm{sec})\end{array}$ \\
\hline Tele-x & 7 & 4.7 & 72 & 31 & 74 & 200 \\
Sham & 5 & 6.2 & 21 & 64 & 34 & 42 \\
Student's $t$ test & $\mathrm{ns}$ & $P<0.04$ & $P<0.04$ & $P<0.04$ & $P<0.02$ \\
\hline
\end{tabular}


TABLE 2

Frequency of Behaviors during the First 10 Min of the 10-Day Spawning Trial

\begin{tabular}{lcccccc}
\hline & & \multicolumn{5}{c}{ Behavior } \\
\cline { 3 - 7 } & $N$ & Approach & $\begin{array}{r}\text { Lateral } \\
\text { display }\end{array}$ & $\begin{array}{r}\text { Frontal } \\
\text { display }\end{array}$ & Quiver & Attack \\
\hline Tele-x & 7 & 0.4 & 8.1 & 0.7 & 1.4 & 0. \\
Sham & 5 & 6.0 & 19.8 & 16.6 & 2.6 & 1.0 \\
Student's $t$ test & & $P<0.001$ & $P<0.03$ & $P<0.002$ & ns & \\
\hline
\end{tabular}

swimming within 2 min following the startle stimulus. In contrast, six of the seven tele-x males remained immobile for more than $3 \mathrm{~min}$ and three did not move during the entire $5-\mathrm{min}$ poststartle period.

\section{Spawning Trial}

The ablation blocked reproduction. All the shams spawned and tended the nest. No tele-x male was seen to spawn or to blow a foamnest, and no eggs were seen in the tank. Some females were observed building a diffuse nest but the male did not participate.

In the initial encounter with the female, males of the tele-x group were less responsive than the shams (Table 2). The tele-x group performed significantly fewer lateral and frontal displays, and they approached the female less frequently. The groups did not differ in the frequency of quivers. The skin color of the tele-x group was dull and pale. The sham group showed the brilliant hues which are typical of intact males engaged in affective display. Within a day, the tele- $x$ males showed avoidance of the female. In some instances, the female attacked the male.

\section{Histology}

In the sham-operated males, the brain showed no damage when examined under a dissecting microscope. The brains of the tele-x males revealed only a stub of tissue protruding in front of the optic chiasm. The histological sections showed that the telencephalic hemispheres, and the olfactory bulbs, were removed on both sides at the level of the anterior commissure (Fig. 4).

\section{DISCUSSION}

The foregoing results show that removal of the telencephalon blocks reproduction but they do not reveal which components of reproduction were 


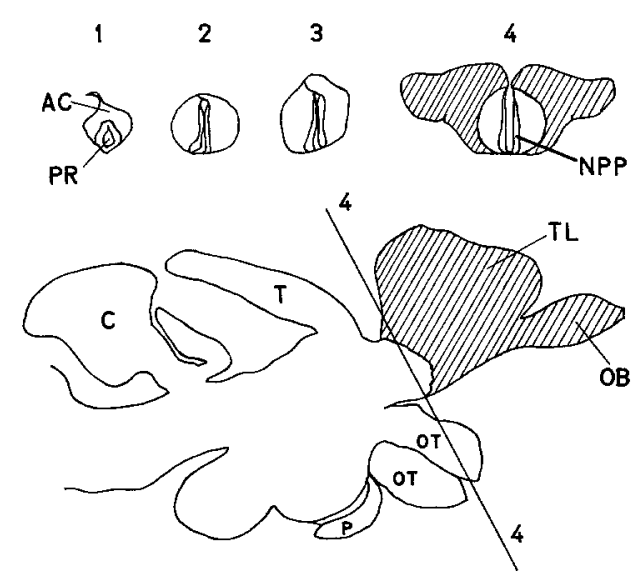

Fig. 4. Diagram of the Macropodus brain. The lesioned areas are cross-hatched. The coronal sections ( 1 to 4 ) are from the tip of the tele-x brain. Section 1 , the most anterior, is at the level of the anterior commissure. Sections 2 to 4 are posterior to the commissure; the torn edges of brain tissues above the preoptic nucleus merged medially. Section 4 is diagrammed over a corresponding section from an intact brain. The approximate plane of section 4 is illustrated by the straight line (4-4) which is drawn through the telencephalon on the diagram of the parasaggital section. $\mathrm{AC}$, anterior commissure; C, cerebellum; NPP, nucleus preopticus periventricularis (Peter and Gill, 1975); $\mathrm{OB}$, olfactory bulb; OT, optic tract; $\mathrm{P}$, pituitary; PR, preoptic recess; T, tectum; TL, telencephalon.

disrupted. Mating depends on a successful courtship. Courtship in Macropodus consists of an exchange of displays in repetitive sequences between the potential mates (Hall, 1968). In our experience, a nest is typically present prior to mating and the final phase of courtship. The lack of nestbuilding in the tele-x male implies that reproduction was blocked earlier in the courtship sequence. The tendency of the tele-x male to avoid or submit to the female also suggests that courtship was disrupted in an early phase. Intact males approach and chase the female. They seldom withdraw or submit when the female approaches or displays. The low display frequency and pale body color of the tele-x males during the first $10 \mathrm{~min}$ of the spawning trial may be linked to their increased startle reactivity. Handling by the investigator and confrontation with a conspecific in the unfamiliar environment could have elicited a conflicting immobility response. Thus the tele-x male's responses to the female may have been inappropriate from the start of the spawning trial. A detailed, quantitative analysis of male behavior during a spawning trial is in progress (Schwagmeyer, Davis, and Kassel, in preparation).

Intact male Macropodus readily build foamnests in the absence of a female or other males. Since tele-x males appear not to blow nests, the 
activation and the coordination of the stereotyped breathing and bubble deposition could be a specific behavioral function of the telencephalon. Other investigators have reported deficits in substrate nestbuilding in the males of various teleosts following telencephalic ablation (Aronson, 1948; Segaar and Nieuwenhuys, 1963; Ribbink, 1972), and Overmier and Gross (1974) proposed that nest digging in Tilapia is controlled by the telencephalon. Demski and Knigge (1971) showed that stimulation near the area dorsalis telencephali pars centralis can elicit nest building in Lepomis macrochirus. Nestbuilding in Macropodus is a distinct pattern of noncommunicative behavior entailing relatively few motor components. In comparison, the behavior in courtship and mating is highly complex. A limited sequence of species typical behavior, such as foamnest building, might be a preferable indicator of behavioral effects in an analysis of the functional anatomy of the telecephalon.

On the basis of results obtained in other fishes showing that retention of positively reinforced operant responding is typically not impaired by telencephalic ablation (Aronson and Herberman, 1960; Savage, 1969), we anticipated only a brief decrease in the swim-through response rate. The behavioral and physiological bases of the low operant rate remain to be investigated. The decrease in responding (Figs. 2 and 3 ) could be due to reduced activity, destruction of memory storage, or a block of memory retrieval. The capacity to relearn the operant response may have been impaired as well. Brief delays in the presentation of food reinforcement resulted in slow learning in tele-x goldfish (Savage, 1969). It is conceivable that in our experiment, the time required for the fish to swim from the manipulandum to the stimulus compartment interfered with reinforcement. However, it is interesting to note that telencephalon ablation did not result in decreased performance of Macropodus in certain food reinforced detour tasks (Warren, 1961). A subsequent investigation in this laboratory showed that substantial recovery of the operant rate can be achieved through extended postsurgery conditioning (Kassel, Davis, and Schwagmeyer,'1976).

The increased reactivity, or sensitivity, to strong stimuli was first indicated by the male's behavior in the home tank within the first few days following surgery. Presentation of food, a tap on the tank wall or movements near the tank typically elicited violent escape movements followed by rigid immobility in tele-x but not sham-operated males. The tele-x males showed increased suppression of air gulp and swimming in the startle trial (Table 1). The increased inhibition of swimming could be the result of the depressed level of prestartle activity of the tele-x males. Though they crossed fewer grid lines during the prestartle period, generally the tele-x males did not impress us as being less active than intact males. The depressed prestartle activity level may have been a response to the handling at the start of the trial. The tendency to freeze when handled was also evident at the start of the operant sessions. The sudden appearance of the conspecific visual stimulus and 
the up-down movement of the door to the stimulus compartment may have elicited startle reactions which interfered with the operant response. Whether the reaction to startle stimuli is decreased concurrently with recovery of operant responding remains to be determined.

Hyperreactivity produced by telencephalic ablation may influence the results of behavioral trials in which the fish is handled, placed in an unfamiliar place, or administered strong stimuli. Tele-x fish may adapt more slowly than control fish, which could mean that interference due to stimulus-induced immobility can be substantially diminished by prolonging the intervals between trials and related treatments. It would be interesting to know whether removal of the telencephalic hemispheres results in increased startle reactions in other teleosts. Ribbink (1972) observed that tele-x cichlids show exaggerated escape in response to movement overhead.

A common view has been that tele-x fish are less and not more reactive to external stimuli. Immobility, reduced spontaneity, long response latency, slow movements in the completion of instrumental responses, and lack of conditioned arousal to conditioned stimuli are frequently reported in investigations of learning performance, social behavior, and nutritive behavior of tele- $x$ teleosts (Hale, 1956; Segaar, 1965; Aronson and Kaplan, 1968; Overmier and Flood, 1969; Savage, 1969; Shapiro, Schuckman, Sussman, and Tucker, 1974). Long latency (etc.) has been attributed to an impairment of neural mechanisms of arousal, or of nonspecific response activation (Aronson and Kaplan, 1968; Aronson, 1970). Regestein (1968) noted that in shock avoidance trials unilateral tele-x goldfish showed shorter response latencies to unilaterally presented stimuli and concluded that the fish were "hyperemotional." The reported sluggishness of (bilateral) tele-x fish was interpreted by Regestein as reflecting a "too high" level of "background arousal." Behavioral effects due to impaired "arousal" could be difficult to distinguish from startle-induced immobility, or slowness stemming from aversion to stimulation. Hyperreactivity and the impairment of learning may be independent effects of telencephalic ablation. It is conceivable that hyperreactivity is more readily detected in some behavioral settings than others; differences in reactivity between intact and lesioned fish may be small in extraordinarily stressful environments. In our view, regulation of responsiveness to external stimuli could be a fundamental function of the teleost telencephalon. Advances in research on the functional anatomy of the telencephalon may be facilitated by further experiments with lesions in local brain areas (e.g., Segaar and Nieuwenhuys, 1963) in various species and by a continuing search for behaviors which strongly reflect telencephalon control. 


\section{REFERENCES}

Aronson, L. R. (1948). Problems in the behavior and physiology of a species of African mouthbreeding fish. Trans. N.Y. Acad. Sci. Ser. 2 33-42.

Aronson, L. R. (1970). Functional evolution of the forebrain in lower vertebrates. In L. R. Aronson, E. Tobach, D. S. Lehrman, and J. S. Rosenblatt (Eds.), "Development and Evolution of Behavior: Essays in Memory of T. C. Schneirla," pp. 75-107. San Francisco: W. H. Freeman.

Aronson, L. R., and Herberman, R. (1960). Persistence of a conditioned response in the cichlid fish, Tilapia macrocephala, after forebrain and cerebellar ablations. Anat. Rec. 138, 332.

Aronson, L. R., and Kaplan, H. (1968). Function of the teleostean forebrain. In D. Ingle (Ed.), "The Central Nervous System and Fish Behavior," pp. 107-125. Chicago: University of Chicago Press.

Bernstein, J. J. (1970). Anatomy and physiology of the central nervous system. In W. S. Hoar and D. J. Randall (Eds.), "Fish Physiology," Vol. 4, pp. 1-90. New York: Academic Press.

Davis, R. E. (1975). Readiness to display in the paradise fish Macropodus opercularis (L.), Belontiidae: The problem of general and specific effects of social isolation. Behav. Biol. 15, 419-433.

Davis, R. E., Harris, C., and Shelby, J. (1974). Sex differences in aggressivity and the effects of social isolation in the anabantoid fish, Macropodus opercularis. Behav. Biol. 11, 497-509.

Davis, R. E., and Kassel, J. (1975). The ontogeny of agonistic behavior and the onset of sexual maturation in the paradise fish, Macropodus opercularis (Linnaeus). Behav. Biol. 14, 31-39.

Davis, R. E., Mitchell, M., and Dolson, L. (1975). The effects of methallibure on conspecific visual reinforcement and social display frequency in the paradise fish, Macropodus opercularis (L.), Belontiidae. Physiol. Behav., in press.

Demski, L. S., and Knigge, K. M. (1971). The telencephalon and hypothalamus of the bluegill (Lepomis macrochirus): Evoked feeding, aggressive and reproductive behavior with representative frontal sections. J. Comp. Neurol. 143, 1-16.

Hale, E. B. (1956). Social facilitation and forebrain function in maze performance of green sunfish, Lepomis cyanellus. Physiol. Zool. 29, 93-106.

Hall, D. D. (1968). A quantitative analysis of courtship and reproductive behavior in the paradise fish, Macropodus opercularis (Linnaeus). Z. Tierpsychol. 25, 834-842.

Healy, E. G. (1957). The nervous system. In M. E. Brown (ed.), "The Physiology of Fishes," Vol. 2, pp. 1-119. New York: Academic Press.

Janzen, W. (1933). Untersuchungen iiber Grosshirn funktionen des goldfisches. Zool. Jahrb. Abt. Allgem. Zool. Physiol. Tiere 52, 591-628.

Kamrin, R. P., and Aronson, L. R. (1954). The effects of forebrain lesions on mating behavior in the male platyfish, Xiphiphorous maculatus. Zoologica (N.Y.) 39, 133-140.

Kassel, J., Davis, R. E., and Schwagmeyer, P. (1976). Telencephalic lesions and behavior in the teleost, Macropodus opercularis: Further analysis of reproductive and operant behavior in the male. Behav. Biol. 18, 179-188.

Liem, K. F. (1963). "The Comparative osteology and phylogeny of the anabantoiedei (Teleostei, Pisces)." Urbana: University of Illinois Press.

Noble, G. K. (1936). Function of the corpus striatum in the social behavior of fishes. Anat. Rec. 64, 34.

Noble, G. K. (1937). Effect of lesions of the corpus striatum on the brooding behavior of cichlid fishes. Anat. Rec. 70, 58. 
Noble, G. K., and Borne, R. (1941). The effect of forebrain lesions on the sexual and fighting behavior of Betta spelndens and other fishes. Anat. Rec. 79, 49.

Overmier, J. B., and Flood, N. B. (1969). Passive avoidance in forebrain ablated teleost fish, Carassius auratus. Physiol. Behav. 4, 791-794.

Overmier, J. B., and Gross, D. (1974). Effects of telencephalic ablation upon nestbuilding and avoidance behaviors in East African mouthbreeding fish, Tilapia mossambica. Behav. Biol. 12, 211-222.

Peter, R. E, and Gill, V. E. (1975). A stereotaxic atlas and technique for forebrain nuclei of the goldfish, Carassius auratus. J. Comp. Neurol. 159, 103-128.

Regestein, Q. R. (1968). Some monocular emotional effects of unilateral hypothalamic lesions in goldfish. In D. Ingle (Ed.), "The Central Nervous System and Fish Behavior," pp. 139-144. Chicago: The University of Chicago Press.

Ribbink, A. J. (1972). The behaviour and brain function of the cichlid fish, Hemihaplochromis philander. Zool. Africana 7, 21-41.

Savage, G. E. (1969). Telencephalic lesions and avoidance behavior in food-reinforced behaviour in the goldfish (Carassius auratus). Anim. Behav. 17, 760-772.

Segaar, J. (1965). Behavioral aspects of degeneration and regeneration in fish brain: A comparison with higher vertebrates. Progr. Brain Res. 14, 143-231.

Segaar, J., and Nieuwenhuys, R. (1963). New ethophysical experiments with male Gasterosteus aculeatus. Anim. Behav. 11, 331-344.

Shapiro, S., Schuckman, H., Sussman, D., and Tucker, A. M. (1974). Effects of telencephalic lesions on the gill cover response of Siamese fighting fish. Physiol. Behav. 13, 749-755.

Warren, J. M. (1961). The effect of telencephalic injuries on learning by paradise fish, Macropodus opercularis. J. Comp. Physiol. Psychol. 54, 130-132. 